\title{
Pigmented spindle cell variant of a thymic atypical carcinoid in an octogenarian
}

\author{
Yasushi Sakamaki ${ }^{1 *} \mathbb{D}$, Ryo Tanaka ${ }^{1}$, Daisuke Ishida ${ }^{1}$, Hiromi Tsuji ${ }^{2}$, Asako Mike $^{2}$ and Hironao Yasuoka ${ }^{2}$
}

\begin{abstract}
Background: A pigmented carcinoid is an extremely rare variant of carcinoid characterized by melanin pigmentation of the tumor, with only five cases described in the literature. In addition, thymic carcinoids are rare in elderly patients and their prognosis after resection of the carcinoid tumor is unclear.

Case presentation: An anterior mediastinal tumor was incidentally found in an 82-year-old man who had been diagnosed with acute thoracic empyema. The tumor was considered most likely to be a noninvasive thymoma or thymic carcinoma for which surgery was indicated after the resolution of the empyema. The tumor was completely resected 4 months after the empyema surgery, and the patient had an uneventful postoperative course. A cut surface of the resected specimen was extensively pigmented and appeared dark-brownish, with abundant melanin pigmentation later confirmed in the spindle-shaped tumor cells. Based on the histologic examination and immunohistochemical study, melanoma was eliminated as a differential diagnosis and the tumor was diagnosed as a pigmented atypical carcinoid of the thymus.
\end{abstract}

Conclusions: This report provides additional knowledge on thymic pigmented carcinoids and thymic atypical carcinoids in elderly patients.

Keywords: Thymic neuroendocrine tumor, Thymic carcinoid, Pigmented carcinoid, Atypical carcinoid, Spindle cell carcinoid

\section{Background}

A pigmented carcinoid (PC) is an extremely rare carcinoid variant. The unique histologic feature of PCs is that the tumor cells contain melanin pigment and show melanocytic differentiation. Herein, we report a case in which a thymic atypical carcinoid with melanin pigmentation was incidentally found as a solitary tumor of the anterior mediastinum in an octogenarian patient.

\section{Case presentation}

An 82-year-old man with no relevant medical history was admitted to our hospital with a diagnosis of acute left thoracic empyema. Computed tomography revealed

\footnotetext{
*Correspondence: sak@serenade.plala.or.jp

${ }^{1}$ Department of Chest Surgery, Osaka Police Hospital, Kitayamacho 10-31,

Tennoji, Osaka 543-0035, Japan

Full list of author information is available at the end of the article
}

a solitary mass measuring $7 \times 5 \times 3 \mathrm{~cm}$ in the anterior mediastinum (Fig. 1a). The computed tomography findings suggested no association between the mass and the empyema. The empyema resolved quickly after thoracoscopic surgery. The patient subsequently underwent further investigation of the mediastinal mass in accordance with the preoperatively provided informed consent. Magnetic resonance imaging revealed a highintensity signal in a large part and a low-intensity signal in the remaining part in the mass on diffusion-weighted images, which confirmed that the lesion was a solid tumor with a liquid component, most likely a high-grade thymoma or thymic carcinoma (Fig. 1b). No other lesions were detected in the metastatic workup. At 4 months after the empyema surgery, thymectomy was performed through a median sternotomy. The tumor was completely resected and appeared well encapsulated, despite a small area of pericardial adhesion that was excised en bloc. 

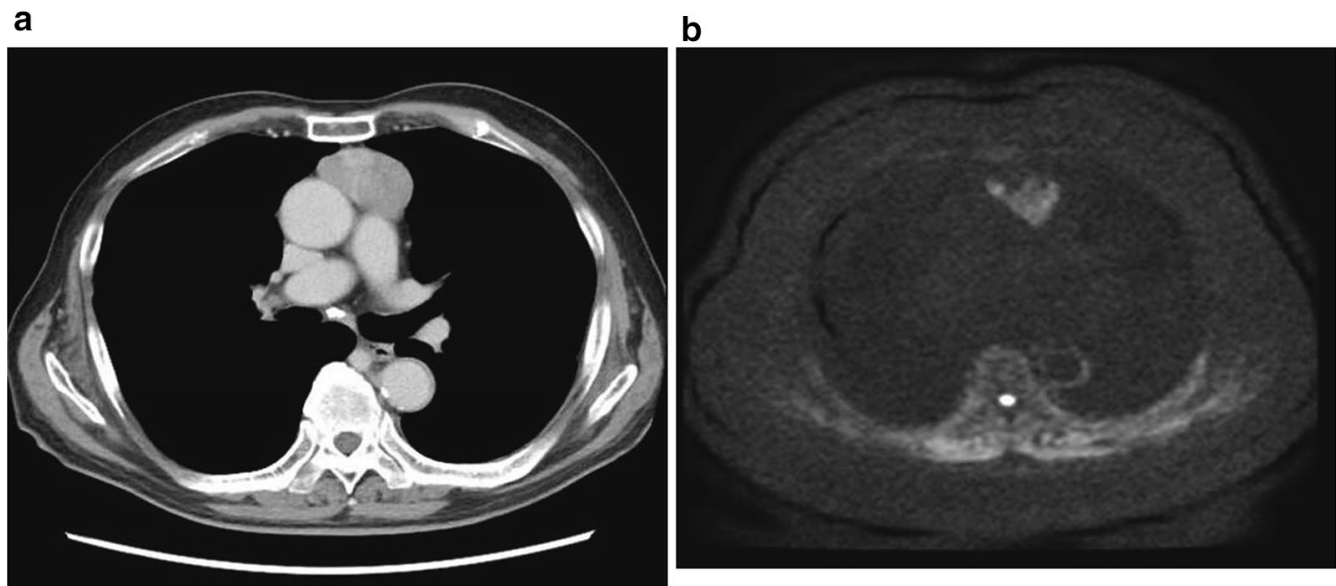

Fig. 1 Tumor appearance on diagnostic imaging. a Computed tomography showing a solitary tumor in the anterior mediastinum. b Diffusion-weighted magnetic resonance image showing a high-intensity signal in the tumor

A cut surface of the resected specimen appeared solid and dark-brownish, suggesting extensive pigmentation of the tumor components (Fig. 2a). Histologic examination revealed a well-circumscribed tumor constituted by organoid or nesting proliferation of spindle-shaped cells with necrosis (Fig. 2b). There were $2-4$ mitotic figures per 10 high-power fields. Dark-brownish pigmentation was easily identifiable in the macrophages and dendritic cells but was less obvious in the tumor cells, reflecting the amount of melanin pigment contained in these cells, as confirmed by Fontana-Masson staining (Fig. 2c). Immunohistochemical study revealed the epithelial nature of the tumor cells based on positive reactivity with cytokeratins AE1/AE3 (Fig. 3a) and CAM5.2, but also revealed their neuroendocrine nature based on positive reactivity with synaptophysin and CD56 (Fig. 3b, c). In addition, there were numerous sustentacular cells surrounding the tumor cell nests, as confirmed by positivity for S-100. The Ki-67 labeling index was $18.7 \%$. Despite positive reactivity of the tumor cells with vimentin, Melan-A, and HMB-45, melanoma was eliminated as a differential diagnosis based on positivity for the two above-mentioned cytokeratin markers and negativity for S-100. The tumor cells also showed negative reactions with epithelial membrane antigen, p63, p40, CD5, CD68, CD57, CD34, smooth muscle actin, CD99, leukocyte common antigen, glial fibrillary acidic protein, and carcinoembryonic antigen. Based on these findings and the opinions of external experts via personal communication, the tumor was finally diagnosed as a pigmented atypical carcinoid of thymic origin. The margins were clear and there was no invasion to the pericardium. However, the tumor had spread to the pericapsular fat tissue and one of the lymph nodes in the perithymic fat (T1aN1M0, stage
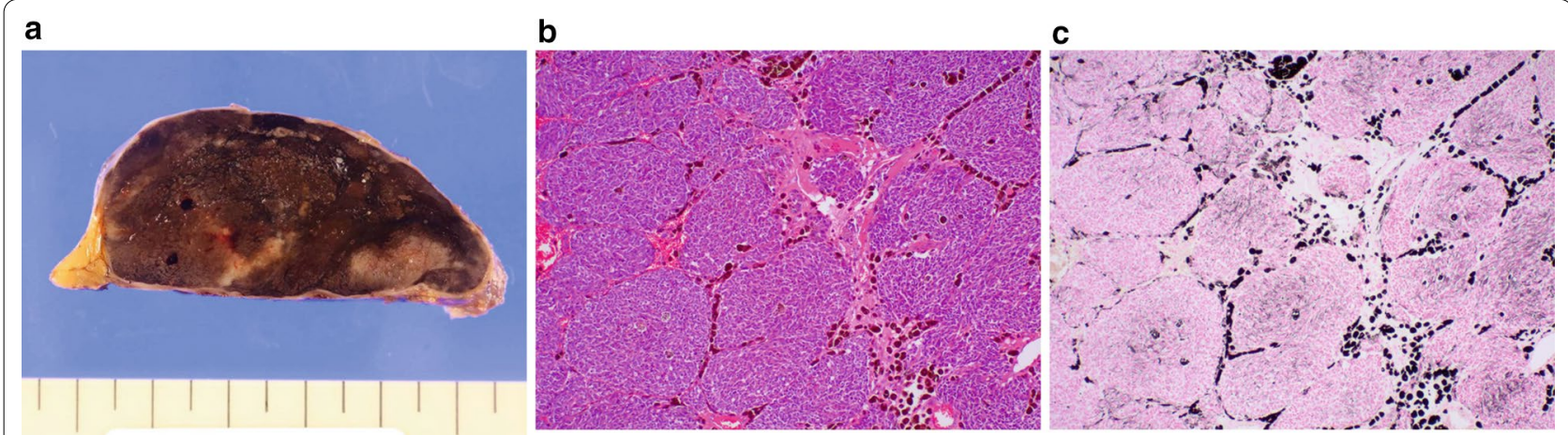

Fig. 2 Gross appearance and histologic findings of the resected tumor. a Dark-brownish cut surface. b Hematoxylin-eosin staining revealing nesting proliferation of the spindle-shaped tumor cells and marked dark-brownish pigmentation in the macrophages. $\mathbf{c}$ Fontana-Masson staining confirming melanin in the pigmented cells 


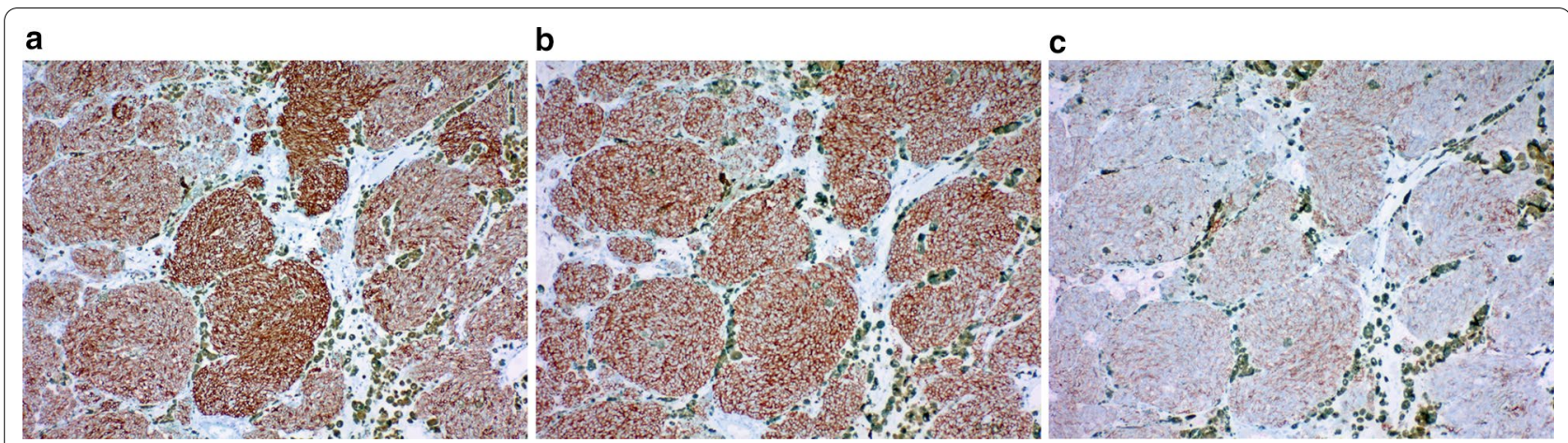

Fig. 3 Immunoreactivity of the tumor cells revealing the epithelial and neuroendocrine nature. a Cytokeratin AE1/AE3. b Synaptophysin. c CD56

IVa). Endocrinology laboratory tests exhibited normal values concerning the adrenal corticoid function. The patient has been doing well without adjuvant therapy. No signs of recurrence have been detected in the 9 months after surgery.

\section{Comments}

Carcinoids are distinctive neuroendocrine tumors that occur most frequently in the gastrointestinal tract, followed by the respiratory tract [1]. Thymic carcinoids are uncommon tumors, accounting for $0.4-2 \%$ of all carcinoids and $2-4 \%$ of all anterior mediastinal tumors $[1,2]$. Rosai and Higa first used the term 'carcinoid tumor of the thymus' to distinguish thymic carcinoids from thymomas based on their microscopic appearance [3]. Recent studies have clarified that carcinoids of the thymus are similar to those of bronchopulmonary origin, ranging in differentiation and behavior from a typical carcinoid to an atypical carcinoid (AC) to small-cell and large-cell carcinomas, despite the differences in genetic alterations between origins [4]. In accordance with the strategy for the classification of tumors in the lung, the World Health Organization currently defines typical carcinoid and AC of the thymus as low-grade and intermediate-grade neuroendocrine tumors, respectively [2].

A PC is an extremely rare variant of carcinoid that has often been reported to originate in the lung, although fewer than ten cases of bronchopulmonary origin have been described in the literature [5]. We found only five case reports of thymic PC to date in an online search of the English literature [6-9]. The reported cases of thymic PC are summarized in Table 1. The histogenesis of thymic PC is currently speculative [9]. Similar to patients with carcinoids, patients with thymic PC are predominantly male $(80 \%)$ and young, ranging in age from 24 to 48 years (median, 46 years) [6-9]. Of these reported cases of thymic PC, two were functioning spindle cell carcinoids with ectopic secretion of adrenocorticotropic hormone $[7,9]$, while the other three were oval or round-to-oval cell carcinoids with no symptoms $[6,8]$. The present case is the first case report of a nonfunctioning spindle cell PC of the thymus as well as that of a thymic PC in an elderly patient.

The prognosis of our patient depends primarily on the aggressiveness of $\mathrm{AC}$, given that melanin pigmentation has no definite prognostic implications [9].

Table 1 Reported cases of pigmented carcinoid of the thymus

\begin{tabular}{|c|c|c|c|c|c|c|c|c|c|}
\hline Case & Author & Age/sex & Symptom & Outcome & Size $(\mathrm{mm})$ & Cell type & $\begin{array}{l}\text { ACTH } \\
\text { secretion }\end{array}$ & Mitosis & Necrosis \\
\hline 1 & $\mathrm{Ho}$ and $\mathrm{Ho}[6]$ & $46 / M$ & None & ND & 110 & Oval & - & ND & + \\
\hline 2 & Lagrange et al. [7] & $48 / F$ & EAS & ND & 80 & Spindle & + & Frequent & + \\
\hline 3 & Klemm et al. [8] & $32 / \mathrm{M}$ & None & NED for 10 years $^{a}$ & $50^{a}$ & Round to oval & - & $3-10 / 10 \mathrm{HPF}$ & + \\
\hline 4 & Klemm et al. [8] & $47 / M$ & None & & & & & & \\
\hline 5 & Kuo [9] & $24 / M$ & EAS & $\begin{array}{l}\text { Recurrence at } \\
17 \text { months postop- } \\
\text { eratively }\end{array}$ & 60 & Spindle & + & Rare & + \\
\hline 6 & Present case & $82 / M$ & None & NED for 9 months & 70 & Spindle & - & $2-4 / 10 \mathrm{HPF}$ & + \\
\hline
\end{tabular}

This table is a revised version of a table in the report by Kuo [9]. Size represents the maximum diameter

ACTH adrenocorticotropic hormone, ND not described, EAS ectopic ACTH syndrome, NED no evidence of disease, HPF high-power field

a Outcome and size only reported for one unspecified case 
Approximately $30 \%$ of ACs metastasize to organs such as the lung, liver, and bone [2]. Although our case has been indolent so far, close observation is needed because of the proven positive lymph node. As thymic carcinoid is a thymic neuroendocrine tumor (TNET), the prognosis of thymic carcinoid may be predicted based on recently reported treatment outcomes and prognostic factors for TNETs [10-13]. These studies reported promising outcomes after surgery, with 5-year overall survival rates ranging from 53 to $84.6 \%$, and confirmed that complete resection is an important prognostic factor and is the optimal treatment for TNETs [10-13]. Concerning adjuvant therapy for TNETs, Filosso et al. [12] reported no statistical advantage in overall survival for adjuvant chemotherapy/radiotherapy based on an analysis of the largest series of TNETs studied to date.

\section{Conclusions}

We treated an octogenarian patient with $\mathrm{PC}$ of thymic origin. $\mathrm{PC}$ is an extremely rare variant of carcinoids and this report provides additional knowledge on mediastinal PCs and thymic atypical carcinoids in elderly patients.

\section{Abbreviations}

PC: Pigmented carcinoid; CD: Cluster of differentiation; HMB: Human melanoma black; AC: Atypical carcinoid; TNET: Thymic neuroendocrine tumor.

\section{Acknowledgements \\ We wish to thank the following experts for their help in the diagnosis of the present case: Dr. Kunimitsu Kawahara, Department of Pathology, Osaka Habikino Medical Center; Dr. Eiichi Konishi, Department of Pathology, Kyoto Prefectural University of Medicine; and Dr. Christopher D. Fletcher, Department of Pathology, Brigham and Women's Hospital and Harvard Medical School. We also thank Kelly Zammit, BVSc, from Edanz Group for editing a draft of this manuscript.}

\section{Authors' contributions}

YS is the corresponding author and carried out the revision of the manuscript. $\mathrm{RT}, \mathrm{DI}, \mathrm{HT}$, and AM carried out the review of the medical record. HY carried out the revision of the manuscript. All authors read and approved the final manuscript.

\section{Funding}

None.

\section{Availability of data and materials}

The authors ensure that all the required datasets are presented in the main manuscript with no additional supporting files.

\section{Ethics approval and consent to participate}

The institutional review board of Osaka Police Hospital approved this case report.

\section{Consent for publication}

Written informed consent was obtained from the patient for publication of this case report and any accompanying images.

\section{Competing interests}

The authors declare that they have no competing interest.

\section{Author details}

${ }^{1}$ Department of Chest Surgery, Osaka Police Hospital, Kitayamacho 10-31, Tennoji, Osaka 543-0035, Japan. ${ }^{2}$ Department of Diagnostic Pathology, Osaka Police Hospital, Osaka, Japan.

Received: 27 October 2020 Accepted: 9 December 2020

Published online: 06 January 2021

\section{References}

1. Yao JC, Hassan M, Phan A, Dagohoy C, Leary C, Mares JE, et al. One hundred years after 'Carcinoid': epidemiology of and prognostic factors for neuroendocrine tumors in 35,825 cases in the United States. J Clin Oncol. 2008:26:3063-72

2. Travis WD, Brambillia E, Burke AP, Marx A, Nicholson AG. World Health Organization classification of tumours of the lung, pleura, thymus and heart. Lyon: IARC Press; 2015.

3. Rosai J, Higa E. Mediastinal endocrine neoplasm of probable thymic origin related to carcinoid tumor. Cancer. 1972;29:1061-74.

4. Ströbel P, Zettl A, Shilo K, Chuang WY, Nicholson AG, Matsuno Y, et al. Tumor genetics and survival of thymic neuroendocrine neoplasms: a multi-institutional clinicopathologic study. Genes Chromosomes Cancer. 2014;53:738-49.

5. Goel A, Addis BJ. Pigmented atypical carcinoid of the lung. Histopathology. 2007;51:263-5.

6. Ho FCS, Ho JCl. Pigmented carcinoid tumor of the thymus. Histopathology. 1977;1:363-9.

7. Lagrange W, Dahm H-H, Karstens J, Feichtinger J, Mittermayer C. Melanocytic neuroendocrine carcinoma of the thymus. Cancer. 1987;59:484-8.

8. Klemm KM, Moran CA, Suster S. Pigmented thymic carcinoids: a clinicopathological and immunohistochemical study of two cases. Mod Pathol. 1999:12:946-8.

9. Kuo T-T. Pigmented spindle cell carcinoid tumor of the thymus with ectopic adrenocorticotropic hormone secretion: report of a rare variant and differential diagnosis of mediastinal spindle cell neoplasms. Histopathology. 2002:40:159-65.

10. Gaur P, Leary C, Yao JC. Thymic neuroendocrine tumors: a SEER database analysis of 160 patients. Ann Surg. 2010;251:1117-21.

11. Cardillo G, Rea F, Lucchi M, Paul MA, Margaritora S, Carleo F, et al. Primary neuroendocrine tumors of the thymus: a multicenter experience of 35 patients. Ann Thorac Surg. 2012;94:241-6.

12. Filosso PL, Yao X, Ahmad U, Zhan Y, Huang J, Ruffini E, et al. Outcome of primary neuroendocrine tumors of the thymus: a joint analysis of the International Thymic Malignancy Interest Group and the European Society of Thoracic Surgeons database. J Thorac Cardiovasc Surg. 2015;149:103-9.

13. Ose N, Maeda H, Inoue M, Mori E, Shintani Y, Matsui H, et al. Results of treatment for thymic neuroendocrine tumors: multicenter clinicopathological study. Interact Cardiovasc Thorac Surg. 2018;26:18-24.

\section{Publisher's Note}

Springer Nature remains neutral with regard to jurisdictional claims in published maps and institutional affiliations. 